\title{
Transversity distributions from difference asymmetries in semi-inclusive DIS
}

\author{
V. Barone, ${ }^{1}$ F. Bradamante, ${ }^{2}$ A. Bressan, ${ }^{2}$ A. Kerbizi, ${ }^{2}$ A. Martin, ${ }^{2}$ A. Moretti, ${ }^{2}$ J. Matousek, ${ }^{2}$ and G. Sbrizzai ${ }^{2}$ \\ ${ }^{1}$ Di.S.I.T., Università del Piemonte Orientale, 15121 Alessandria, Italy, \\ and INFN, Sezione di Torino, 10125 Torino, Italy \\ ${ }^{2}$ Dipartimento di Fisica, Università di Trieste, and INFN, Sezione di Trieste, 34127 Trieste, Italy
}

(Received 25 March 2019; published 5 June 2019)

\begin{abstract}
In recent years information on the transversity distribution $h_{1}$ has been obtained by combining the Collins asymmetry results from semi-inclusive deep inelastic scattering (SIDIS) data on transversely polarized nucleon targets with the information on the fragmentation function of a transversely polarized quark from the asymmetries measured in $e^{+} e^{-}$annihilation into hadrons. An alternative method was proposed a long time ago, which does not require the $e^{+} e^{-}$data but allows one to get ratios of the $u$ and $d$ quark transversity distributions from the SIDIS data alone. The method utilizes the ratio of the difference of the Collins asymmetries of positively and negatively charged hadrons produced on transversely polarized proton and deuteron targets. We have applied this method to the COMPASS proton and deuteron data and extracted the ratio $h_{1}^{d_{v}} / h_{1}^{u_{v}}$. The results are compared to those obtained in a previous point-by-point extraction based both on SIDIS and $e^{+} e^{-}$data.
\end{abstract}

DOI: 10.1103/PhysRevD.99.114004

\section{INTRODUCTION}

Much interest has been dedicated to the transversity distribution in the past 20 years. Usually called $h_{1}$, it is a leading-twist parton distribution function (PDF) which describes the transverse polarization of quarks inside a transversely polarized nucleon (for reviews, see [1-3]).

Being chirally odd, transversity cannot be measured in deep inelastic scattering (DIS). Over the last decade singlespin asymmetries clearly related to the transversity distribution function have been measured in semi-inclusive deep inelastic scattering (SIDIS) on transversely polarized nucleons, namely, in DIS processes in which at least one hadron of the current jet is detected. In these processes the cross section exhibits a spin-dependent azimuthal modulation that can be expressed in terms of a convolution of the transversity PDF and a fragmentation function (FF) which is also chiral odd, thus guaranteeing the cross section to be chirally even. Two observables have been studied so far. The first one is single hadron spin asymmetry, namely, the amplitude of the target-spin-dependent azimuthal modulation of each of the produced hadrons. The second one is the amplitude of the target spin-dependent azimuthal modulation of the plane defined by any two of the oppositely

Published by the American Physical Society under the terms of the Creative Commons Attribution 4.0 International license. Further distribution of this work must maintain attribution to the author(s) and the published article's title, journal citation, and DOI. Funded by SCOAP ${ }^{3}$. charged hadrons produced in the same SIDIS event. In the case of transversely polarized proton targets, clear nonzero azimuthal modulations have been measured for both observables by the HERMES [4,5] and the COMPASS $[6,7]$ Collaborations, assessing beyond any doubt that the transversity PDFs, the single hadron FF, and the dihadron FF are all nonzero. Corresponding measurements on a transversely polarized deuteron target by the COMPASS Collaboration [8,9] gave asymmetries compatible with zero, which have been interpreted as evidence of cancellation between $h_{1}^{u}$ and $h_{1}^{d}$.

The underlying physics of these processes $[10,11]$ is the left-right asymmetry in the hadronization of a transversely polarized quark, where left and right are relative to the plane defined by the quark direction of motion and its transverse spin. Such asymmetry is encoded, in the first case, in the so-called Collins FF $H_{1}^{\perp}$ [12] and, in the second case, in the dihadron FF $H_{1}^{\varangle}[13,14]$. Independent evidence that both the Collins function and the dihadron FF are different from zero came from the measurements of azimuthal asymmetries in hadron inclusive production in $e^{+} e^{-}$annihilation by the Belle [15,16], BABAR [17], and BESIII [18] Collaborations.

Combining the SIDIS data and the $e^{+} e^{-} \rightarrow$ hadrons measurements, first extractions of both the transversity functions and of the two transversely polarized quark FFs have been possible $[19,20]$. In all those works, in order to solve the convolution over the transverse momenta between the transversity PDF and the FF which appears in the cross 
section, some parametrization for both $h_{1}$ and for the FFs had to be assumed. An exception is the recent extraction of transversity [21] which has been performed point by point directly from the COMPASS SIDIS and the Belle $e^{+} e^{-} \rightarrow$ hadrons data, without using any parametrization for the collinear variables.

An alternative way to measure transversity from the Collins asymmetries alone is via the so-called "difference asymmetries," which allow extracting combinations of the $u$ and $d$ quark transversity without knowing the Collins FF. This method was proposed a long time ago [22-24] to access the helicity PDFs and has been used by the SMC Collaboration [25]. It was also included in the COMPASS proposal [26] to measure both longitudinal and transverse spin asymmetries. At that time it looked particularly interesting since the Collins FF was completely unknown. Later it was used to measure the helicity PDFs in COMPASS [27], and recently it was proposed again in the context of the Sivers, Boer-Mulders, and transversity distributions [28]. In the present work the difference asymmetries are used for the first time to access transversity with the COMPASS measurements of the Collins asymmetries on $p$ [6] and $d$ targets [8].

The paper is organized as follows. In Sec. II we describe the formalism and the procedure. Section III is dedicated to the Monte Carlo studies. The results are discussed in Sec. IV.

\section{CROSS SECTIONS AND DIFFERENCE ASYMMETRIES}

In this paper we extract the asymmetries of differences from the Collins asymmetries measured by the COMPASS Collaboration impinging a $160 \mathrm{GeV} / \mathrm{c}$ momentum muon beam either on a transversely polarized deuteron $\left({ }^{6} \mathrm{LiD}\right)$ target or a transversely polarized proton $\left(\mathrm{NH}_{3}\right)$ target. The data we have considered were taken in the years 2002-2004 [8] and 2010 [6].

In order to ensure the DIS regime, only events with photon virtuality $Q^{2}>1(\mathrm{GeV} / \mathrm{c})^{2}$, fractional energy of the virtual photon $0.1<y<0.9$, and mass of the hadronic final state system $W>5 \mathrm{GeV} / \mathrm{c}^{2}$ were considered in the data analysis. The charged hadrons were required to have at least $0.1 \mathrm{GeV} / \mathrm{c}$ transverse momentum $P_{T}$ with respect to the virtual photon direction and a fraction of the available energy $z>0.2$. All the details of the event selection and of the analysis can be found in $[6,8]$. The published data are binned in $x$, the target nucleon momentum fraction carried by the struck quark, in $z$ and in $P_{T}$. In our analysis we only consider the asymmetry data binned in $x$, in 9 bins, from 0.003 to 0.7 .

In the following, for simplicity, we write explicitly only the Collins part of the SIDIS transverse spin-dependent cross section and consider charged pions, even if, at the end, we use the results for charged hadrons assuming they are all pions, as was done, for instance, in [21]. This is justified by the fact that the hadron samples are mainly constituted of pions (about 70\% for positive hadrons, $75 \%$ for negative hadrons), a composition which is almost independent of $x[6]$.

The SIDIS cross section can be written as

$$
\sigma_{t}^{ \pm}\left(\Phi_{C}\right)=\sigma_{0, t}^{ \pm}+f P_{t} D_{N N} \sigma_{C, t}^{ \pm} \sin \Phi_{C}
$$

where $\Phi_{C}$ is the Collins angle, $f$ is the target dilution factor, $P_{t}$ is the target polarization, and $D_{N N}$ is the mean transverse spin-transfer coefficient not included in $\sigma_{C}$ to simplify the expressions used in the following. Only the deuteron (or hydrogen) nuclei in the targets are polarized, and the target dilution factor $f$ is given by the ratio of the absorption cross sections on the deuteron (or proton) to that of all nuclei in the target. The signs \pm refer to the pion charge and $t=p, d$ is the target type. The Collins angle $\Phi_{C}=\phi_{h}+\phi_{S}-\pi$ is the sum of the azimuthal angles $\phi_{h}$ of the hadron transverse momentum and of the spin direction $\phi_{S}$ of the target nucleon with respect to the lepton scattering plane, in a reference system in which the $z$ axis is the virtual photon direction.

We take as a measure of the Collins asymmetry the ratio

$$
A_{C, t}^{ \pm}=\frac{\sigma_{C, t}^{ \pm}}{\sigma_{0, t}^{ \pm}}
$$

In terms of the ordinary PDFs and FFs, the unpolarized part of the cross sections in Eq. (1) can be written as (omitting a kinematic factor that cancels out when taking the ratios of cross sections)

$$
\begin{gathered}
\sigma_{0, p}^{+} \sim x\left[\left(4 f_{1}^{u}+f_{1}^{\bar{d}}\right) D_{1, \mathrm{fav}}+\left(4 f_{1}^{\bar{u}}+f_{1}^{d}\right) D_{1, \mathrm{unf}}\right. \\
\left.+\left(f_{1}^{s}+f_{1}^{\bar{s}}\right) D_{1, s}\right], \\
\sigma_{0, p}^{-} \sim x\left[\left(4 f_{1}^{u}+f_{1}^{\bar{d}}\right) D_{1, \mathrm{unf}}+\left(4 f_{1}^{\bar{u}}+f_{1}^{d}\right) D_{1, \mathrm{fav}}\right. \\
\left.\quad+\left(f_{1}^{s}+f_{1}^{\bar{s}}\right) D_{1, s}\right], \\
\sigma_{0, d}^{+} \sim x\left[\left(f_{1}^{u}+f_{1}^{d}\right)\left(4 D_{1, \mathrm{fav}}+D_{1, \mathrm{unf}}\right)\right. \\
\left.+\left(f_{1}^{\bar{u}}+f_{1}^{\bar{d}}\right)\left(D_{1, \mathrm{fav}}+4 D_{1, \mathrm{unf}}\right)+2\left(f_{1}^{s}+f_{1}^{\bar{s}}\right) D_{1, s}\right], \\
\sigma_{0, d}^{-} \sim \\
\quad x\left[\left(f_{1}^{u}+f_{1}^{d}\right)\left(D_{1, \mathrm{fav}}+4 D_{1, \mathrm{unf}}\right)\right. \\
\left.+\left(f_{1}^{\bar{u}}+f_{1}^{\bar{d}}\right)\left(4 D_{1, \mathrm{fav}}+D_{1, \mathrm{unf}}\right)+2\left(f_{1}^{s}+f_{1}^{\bar{s}}\right) D_{1, s}\right],
\end{gathered}
$$

where $D_{1, \text { fav }}\left(D_{1, \text { unf }}\right)$ is the favored (unfavored) unpolarized FF, $D_{1, s}$ is the strange sea unpolarized FF, and $f_{1}^{q}$ are the unpolarized PDFs.

Following [21], the corresponding spin-dependent cross sections are obtained by replacing $f_{1}^{q}$ with the transversity 
PDFs $h_{1}^{q}$ and the FFs $D_{1}$ with the "half moments" of the Collins function, $H_{1}^{\perp(1 / 2)}$, defined as

$$
H_{1}^{\perp(1 / 2)}\left(z, Q^{2}\right) \equiv \int \mathrm{d}^{2} \boldsymbol{p}_{T} \frac{p_{T}}{z M_{h}} H_{1}^{\perp}\left(z, p_{T}^{2}, Q^{2}\right) .
$$

Thus, we have

$$
\begin{array}{r}
\sigma_{C, p}^{+} \sim x\left[\left(4 h_{1}^{u}+h_{1}^{\bar{d}}\right) H_{1, \mathrm{fav}}^{\perp(1 / 2)}+\left(4 h_{1}^{\bar{u}}+h_{1}^{d}\right) H_{1, \mathrm{unf}}^{\perp(1 / 2)}\right], \\
\sigma_{C, p}^{-} \sim x\left[\left(4 h_{1}^{u}+h_{1}^{\bar{d}}\right) H_{1, \mathrm{unf}}^{\perp(1 / 2)}+\left(4 h_{1}^{\bar{u}}+h_{1}^{d}\right) H_{1, \mathrm{fav}}^{\perp(1 / 2)}\right], \\
\sigma_{C, d}^{+} \sim x\left[\left(h_{1}^{u}+h_{1}^{d}\right)\left(4 H_{1, \mathrm{fav}}^{\perp(1 / 2)}+H_{1, \mathrm{unf}}^{\perp(1 / 2)}\right)\right. \\
\left.+\left(h_{1}^{\bar{u}}+h_{1}^{\bar{d}}\right)\left(H_{1, \mathrm{fav}}^{\perp(1 / 2)}+4 H_{1, \mathrm{unf}}^{\perp(1 / 2)}\right)\right], \\
\sigma_{C, d}^{-} \sim x\left[\left(h_{1}^{u}+h_{1}^{d}\right)\left(H_{1, \mathrm{fav}}^{\perp(1 / 2)}+4 H_{1, \mathrm{unf}}^{\perp(1 / 2)}\right)\right. \\
\left.+\left(h_{1}^{\bar{u}}+h_{1}^{\bar{d}}\right)\left(4 H_{1, \mathrm{fav}}^{\perp(1 / 2)}+H_{1, \mathrm{unf}}^{\perp(1 / 2)}\right)\right],
\end{array}
$$

where we have assumed $H_{1, s}^{\perp(1 / 2)}=0$.
We now define the difference asymmetries as

$$
A_{D, t}=\frac{\sigma_{C, t}^{+}-\sigma_{C, t}^{-}}{\sigma_{0, t}^{+}+\sigma_{0, t}^{-}}
$$

In [23] an alternative definition was proposed, namely,

$$
A_{D, t}^{\prime}=\frac{\sigma_{C, t}^{+}-\sigma_{C, t}^{-}}{\sigma_{0, t}^{+}-\sigma_{0, t}^{-}}
$$

As we will see, the two definitions give the same results. For the sake of simplicity, our discussion in the following is centered on the definition (12), but we also briefly summarize the results obtained with Eq. (13).

Writing explicitly the asymmetries, one gets

$$
\begin{aligned}
A_{D, p} & =\frac{1}{9} \frac{H_{1, \mathrm{fav}}^{\perp(1 / 2)}-H_{1, \mathrm{unf}}^{\perp(1 / 2)}}{\sigma_{0, p}^{+}+\sigma_{0, p}^{-}}\left(4 h_{1}^{u_{v}}-h_{1}^{d_{v}}\right), \\
A_{D, d} & =\frac{1}{3} \frac{H_{1, \mathrm{fav}}^{\perp(1 / 2)}-H_{1, \mathrm{unf}}^{\perp(1 / 2)}}{\sigma_{0, d}^{+}+\sigma_{0, d}^{-}}\left(h_{1}^{u_{v}}+h_{1}^{d_{v}}\right) .
\end{aligned}
$$

When taking the ratios of the asymmetries on the deuteron and proton, the Collins FFs cancel out:

$$
\frac{A_{D, d}}{A_{D, p}}=3\left[\frac{\left(4 f_{1}^{u}+4 f_{1}^{\bar{u}}+f_{1}^{d}+f_{1}^{\bar{d}}\right)\left(D_{1, \mathrm{fav}}+D_{1, \mathrm{unf}}\right)+2\left(f_{1}^{s}+f_{1}^{\bar{s}}\right) D_{1, s}}{5\left(f_{1}^{u}+f_{1}^{d}+f_{1}^{\bar{u}}+f_{1}^{\bar{d}}\right)\left(D_{1, \mathrm{fav}}+D_{1, \mathrm{unf}}\right)+4\left(f_{1}^{s}+f_{1}^{\bar{s}}\right) D_{1, s}}\right] \frac{h_{1}^{u_{v}}+h_{1}^{d_{v}}}{4 h_{1}^{u_{v}}-h_{1}^{d_{v}}}
$$

and the only unknowns are the transversity PDFs. Thus, by measuring $A_{D}$ on $p$ and $d$, one obtains the ratio $h_{1}^{d_{v}} / h_{1}^{u_{v}}$ in terms of known quantities.

In order to determine $A_{D, t}$, one should, in principle, fit the quantity

$$
\sigma_{t}^{D}\left(\Phi_{C}\right)=\left(\sigma_{0, t}^{+}-\sigma_{0, t}^{-}\right)+f P_{t} D_{N N}\left(\sigma_{C, t}^{+}-\sigma_{C, t}^{-}\right) \sin \Phi_{C}
$$

and extract the amplitude of the $\sin \Phi_{C}$ modulation. Since the acceptances for positively and negatively charged particles are not usually the same, one should correct the number of events for the acceptance before taking the differences and treat the statistical errors carefully.

The measurements are much simpler if the $\Phi_{C}$ acceptance for positively charged particles is equal to that for negatively charged ones. In this case it is not necessary to evaluate the difference asymmetries from the amplitude of the modulation, as it is possible to get them from the measured Collins asymmetries. In fact, one has

$$
A_{D, t}=\frac{\sigma_{0, t}^{+}}{\sigma_{0, t}^{+}+\sigma_{0, t}^{-}} A_{C, t}^{+}-\frac{\sigma_{0, t}^{-}}{\sigma_{0, t}^{+}+\sigma_{0, t}^{-}} A_{C, t}^{-},
$$

where the ratios of the cross sections are known. In order to apply this procedure, extensive Monte Carlo studies have been performed. They are described in the next section.

Notice that if one uses instead the definition (13), the ratio of the difference asymmetries has the form

$$
\frac{A_{D, d}^{\prime}}{A_{D, p}^{\prime}}=\frac{4 f_{1}^{u_{v}}-f_{1}^{d_{v}}}{f_{1}^{u_{v}}+f_{1}^{d_{v}}} \frac{h_{1}^{u_{v}}+h_{1}^{d_{v}}}{4 h_{1}^{u_{v}}-h_{1}^{d_{v}}}
$$

and the equivalent of Eq. (18) is

$$
A_{D, t}^{\prime}=\frac{\sigma_{0, t}^{+}}{\sigma_{0, t}^{+}-\sigma_{0, t}^{-}} A_{C, t}^{+}-\frac{\sigma_{0, t}^{-}}{\sigma_{0, t}^{+}-\sigma_{0, t}^{-}} A_{C, t}^{-} .
$$

\section{MONTE CARLO STUDIES}

The acceptance of the COMPASS spectrometer for positively charged and negatively charged hadrons has been investigated with Monte Carlo simulations. In the case of the deuteron data, collected in the years 2002-2004, this work was a prerequisite to the extraction of the $\sin \phi_{h}$, $\cos \phi_{h}$, and $\cos 2 \phi_{h}$ modulations [29] which are expected in 

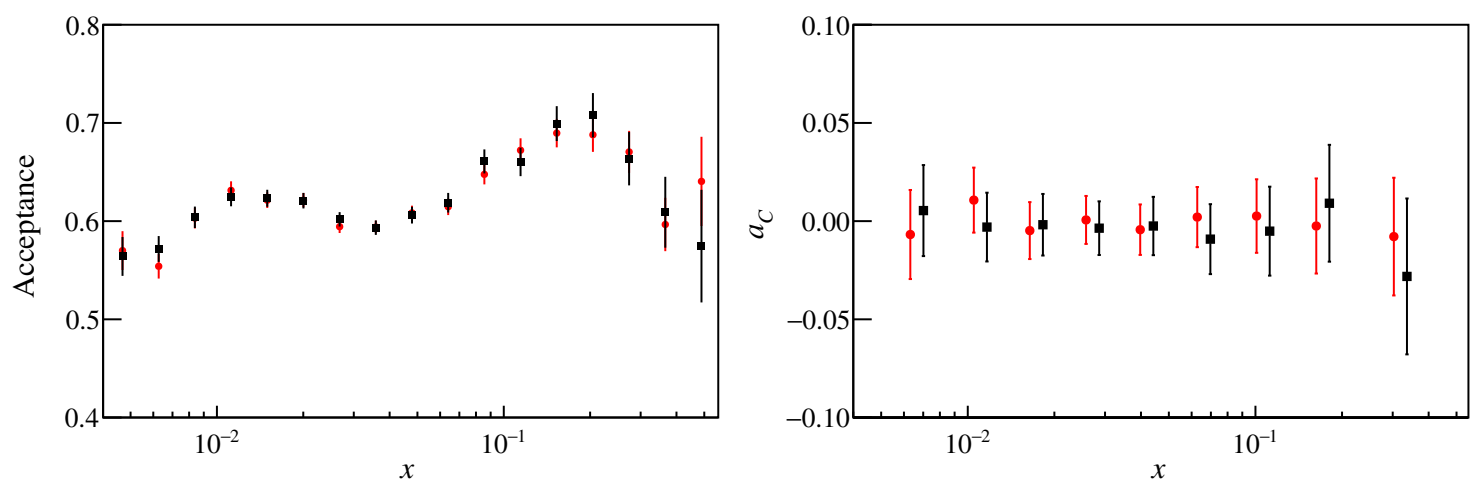

FIG. 1. Left panel: Experimental acceptance for positively charged (red points) and negatively charged (black points) hadrons as a function of $x$. Right panel: Amplitude of the $a_{C}$ modulation in the azimuthal acceptance as a function of $x$ for positively (red points) and negatively (black squares) charged hadrons.

the unpolarized SIDIS cross section. Within the statistical errors, the acceptance is essentially the same for positive and negative hadrons. In 2010, when the proton data were collected, the spectrometer was substantially different from the one utilized for the deuteron data taking; thus the whole work had to be repeated. To this end we have used a full Monte Carlo chain with LEPTO [30] as an event generator and TGEANT, a GEANT4 [31] based program, for the simulation of the particle interaction with the COMPASS apparatus and the detector response. The Monte Carlo events have been reconstructed with the COMPASS package CORAL [32] and analyzed to extract the acceptances and the acceptance ratios. The same kinematic selections used for the analysis of the real data have been applied on the generated variables and on the reconstructed ones. While integrating over the other kinematical variables, the acceptances $\mathcal{A}(x)=N^{\mathrm{rec}}(x) / N^{\mathrm{gen}}\left(x^{\mathrm{gen}}\right)$ have been obtained by taking the ratio of the reconstructed and generated events counted in every bin using, respectively, the generated $x^{\text {gen }}$ and the reconstructed $x^{\text {rec }}$ values. In this way the smearing due to the experimental resolution is also accounted for.

The acceptances, which include both the geometrical acceptance of the apparatus and the reconstruction efficiency, are shown in Fig. 1 (left panel). The acceptances for positively (red points) and negatively (black points) charged hadrons are in good agreement, and the small differences are compatible with the statistical fluctuations. Their ratios are constant over the full $x$ range of the measurement, with an average value of $1.003 \pm 0.006$.

A possible Collins modulation in the acceptance was also studied, separately for positively and negatively charged hadrons, by fitting in each $x$ bin the $\Phi_{C}$ distribution with a function $c\left(1+a_{C} \sin \left(\Phi_{C}\right)\right)$. The results for $a_{C}$ are shown in Fig. 1 (right panel). The amplitudes of the modulation are compatible with zero over the full $x$ range for both positive and negative hadrons. This result also stays true when repeating the procedure for the ratio of the acceptances.

\section{RESULTS}

On the basis of the Monte Carlo results, the difference asymmetries have been calculated using Eq. (18) with the Collins asymmetries from the 2010 COMPASS data. Actually, since $\sigma_{0, t}^{ \pm} \sim N_{t}^{ \pm}$and $\operatorname{var}\left(A_{C, t}^{ \pm}\right) \sim 1 / N_{t}^{ \pm}$, where $N_{t}^{ \pm}$is the total number of hadrons that have been used to extract the Collins asymmetries, in a given $x$ bin, Eq. (18) can be rewritten as

$$
\begin{aligned}
A_{D, t}= & \frac{\operatorname{var}\left(A_{C, t}^{-}\right)}{\operatorname{var}\left(A_{C, t}^{+}\right)+\operatorname{var}\left(A_{C, t}^{-}\right)} A_{C, t}^{+} \\
& -\frac{\operatorname{var}\left(A_{C, t}^{+}\right)}{\operatorname{var}\left(A_{C, t}^{+}\right)+\operatorname{var}\left(A_{C, t}^{-}\right)} A_{C, t}^{-} .
\end{aligned}
$$

The calculation of the difference asymmetries can thus be performed using the published COMPASS data for $A_{C, t}^{ \pm}$and their statistical uncertainties [6]. An interesting remark is that $A_{D, t}$ is equal to the weighted mean of the Collins asymmetries for positive and negative hadrons, after changing the sign to $A_{C, t}^{-}$. The results for the proton and the deuteron are shown in Fig. 2.

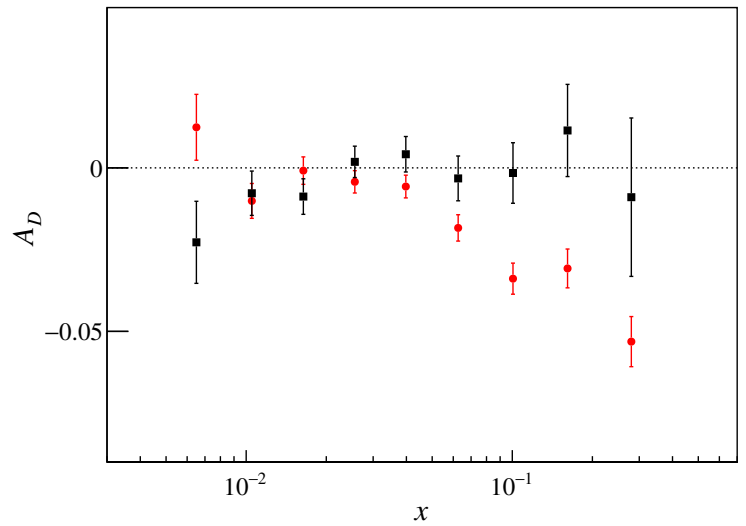

FIG. 2. Difference asymmetries $A_{D, p}$ (red points) and $A_{D, d}$ (black points) as a function of $x$. 


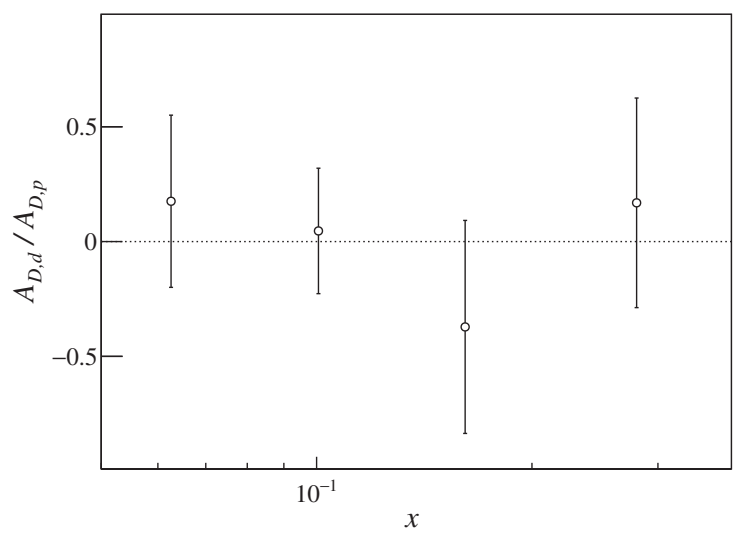

FIG. 3. Ratio $A_{D, d} / A_{D, p}$ of the difference asymmetries on the deuteron and on the proton as a function of $x$. Here and in the next figure, the ratio in the missing $x$ bins has values out of scale with very large statistical errors.

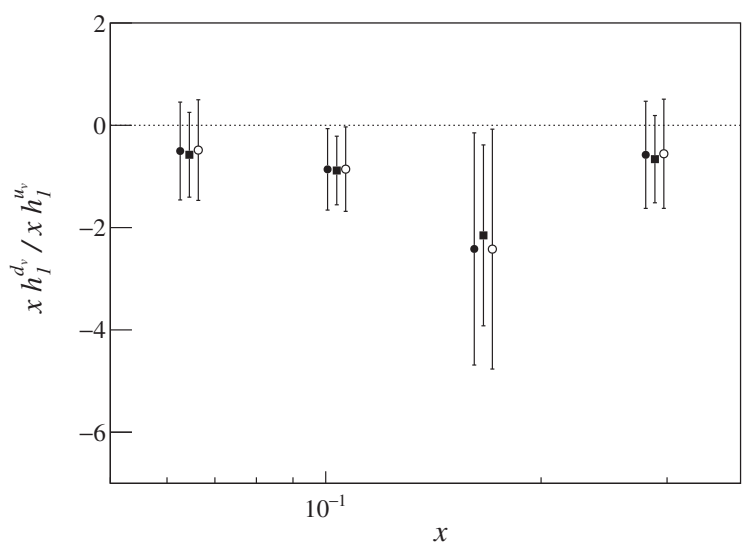

FIG. 4. Ratio $h_{1}^{d_{v}} / h_{1}^{u_{v}}$ from the asymmetries $A_{D}$ (closed circles), from the asymmetries $A_{D}^{\prime}$ (closed squares), and from [21] (open circles).

The ratio $A_{D, d} / A_{D, p}$ is shown in Fig. 3. Only the four points at larger $x$ are plotted in the figure. The points at smaller $x$ have much-too-large uncertainties since the proton asymmetries in that region are compatible with zero.

From the ratios $A_{D, d} / A_{D, p}$ the quantities $\left(h_{1}^{u_{v}}+h_{1}^{d_{v}}\right) /$ $\left(4 h_{1}^{u_{v}}-h_{1}^{d_{v}}\right)$ have been extracted using Eq. (16) and standard parametrizations and tables for the unpolarized PDFs [33] and FFs [34].

Finally, from the quantities $\left(h_{1}^{u_{v}}+h_{1}^{d_{v}}\right) /\left(4 h_{1}^{u_{v}}-h_{1}^{d_{v}}\right)$ the ratios $h_{1}^{d_{v}} / h_{1}^{u_{v}}$ are determined. They are shown as closed circles in Fig. 4. Again, the values in the first five $x$ bins have very large uncertainties, are compatible with zero, and are not plotted in the figure. At larger $x$ the values are negative, in agreement with previous extractions. The same procedure has been carried out starting from the difference asymmetries $A_{D, t}^{\prime}$ and using Eq. (19), getting essentially the same values and similar statistical uncertainties, which are shown as closed squares in Fig. 4. In the same figure we also compare our results with the values of $h_{1}^{d_{v}} / h_{1}^{u_{v}}$ calculated from the transversity values obtained in [21] (open circles). In the evaluation of the uncertainty of the ratio $h_{1}^{d_{v}} / h_{1}^{u_{v}}$ from [21], we take a proper account of the correlations between the extracted values of $h_{1}^{u_{v}}$ and $h_{1}^{d_{v}}$, and we make use of the correlation coefficients as evaluated in [35]. The results of the three determinations are in very good agreement, but some reduction (up to $20 \%$ ) of the uncertainties can be observed in the ratios obtained in the present work from the difference asymmetries $A_{D, t}^{\prime}$.

\section{CONCLUSION}

We have determined for the first time the transverse-spin difference asymmetries of positively and negatively charged hadrons using the SIDIS $p$ and $d$ COMPASS data. Thanks to the good COMPASS spectrometer acceptance, they could be easily obtained from the measured Collins asymmetries. From the ratio between the difference asymmetries on deuterons and on protons, we have extracted the quantity $h_{1}^{d_{v}} / h_{1}^{u_{v}}$, the ratio between the valence $d$-quark and $u$-quark transversity PDF.

At small $x$ the difference asymmetries on the protons are compatible with zero; thus the statistical uncertainty on the ratio $h_{1}^{d_{v}} / h_{1}^{u_{v}}$ is too large, and no useful information is provided by the present analysis. On the other hand, for larger $x(x \geq 0.05)$ the extracted ratio $h_{1}^{d_{v}} / h_{1}^{u_{v}}$ has negative sign and is in very good agreement with the results of a previous point-by-point extraction.

The method we applied is interesting and simple, and it does not require any knowledge of the Collins fragmentation functions. Hence it strengthens the validity of the methods utilized so far to extract the transversity distributions, based on a combined analysis of SIDIS and $e^{+} e^{-}$ data, and it can be used as a useful cross-check for more elaborated extractions.

\section{ACKNOWLEDGMENTS}

This work is possible thanks to Project No. FRA2015, supported by the Università degli Studi di Trieste. V. B. is partially supported by "Fondi di Ricerca Locale ex-60\%" of the University of Piemonte Orientale. We would like to thank E. Christova and E. Leader for interesting discussions. We are grateful to the COMPASS Collaboration for use of the Monte Carlo chain. 
[1] V. Barone, F. Bradamante, and A. Martin, Prog. Part. Nucl. Phys. 65, 267 (2010).

[2] C. A. Aidala, S. D. Bass, D. Hasch, and G. K. Mallot, Rev. Mod. Phys. 85, 655 (2013).

[3] H. Avakian, A. Bressan, and M. Contalbrigo, Eur. Phys. J. A 52, 150 (2016); 52, 165(E) (2016).

[4] A. Airapetian et al. (HERMES Collaboration), Phys. Lett. B 693, 11 (2010).

[5] A. Airapetian et al. (HERMES Collaboration), J. High Energy Phys. 06 (2008) 017.

[6] C. Adolph et al. (COMPASS Collaboration), Phys. Lett. B 717, 376 (2012).

[7] C. Adolph et al. (COMPASS Collaboration), Phys. Lett. B 736, 124 (2014).

[8] E. S. Ageev et al. (COMPASS Collaboration), Nucl. Phys. B765, 31 (2007).

[9] C. Adolph et al. (COMPASS Collaboration), Phys. Lett. B 713, 10 (2012).

[10] C. Adolph et al. (COMPASS Collaboration), Phys. Lett. B 753, 406 (2016).

[11] A. Kerbizi, X. Artru, Z. Belghobsi, F. Bradamante, and A. Martin, Phys. Rev. D 97, 074010 (2018).

[12] J. C. Collins, Nucl. Phys. B396, 161 (1993).

[13] J. C. Collins, S. F. Heppelmann, and G. A. Ladinsky, Nucl. Phys. B420, 565 (1994).

[14] M. Radici, R. Jakob, and A. Bianconi, Phys. Rev. D 65, 074031 (2002).

[15] R. Seidl et al. (Belle Collaboration), Phys. Rev. D 78, 032011 (2008); 86, 039905(E) (2012).

[16] A. Vossen et al. (Belle Collaboration), Phys. Rev. Lett. 107, 072004 (2011).

[17] J. P. Lees et al. (BABAR Collaboration), Phys. Rev. D 90, 052003 (2014).

[18] M. Ablikim et al. (BESIII Collaboration), Phys. Rev. Lett. 116, 042001 (2016).

[19] M. Anselmino, M. Boglione, U. D’Alesio, J. O. Gonzalez Hernandez, S. Melis, F. Murgia, and A. Prokudin, Phys. Rev. D 92, 114023 (2015) and references therein.
[20] Z. B. Kang, A. Prokudin, P. Sun, and F. Yuan, Phys. Rev. D 93, 014009 (2016) and references therein.

[21] A. Martin, F. Bradamante, and V. Barone, Phys. Rev. D 91, 014034 (2015).

[22] L. L. Frankfurt, M. I. Strikman, L. Mankiewicz, A. Schäfer, E. Rondio, A. Sandacz, and V. Papavassiliou, Phys. Lett. B 230, 141 (1989).

[23] E. Christova and E. Leader, Nucl. Phys. B607, 369 (2001).

[24] A. N. Sissakian, O. Yu. Shevchenko, and O. N. Ivanov, Phys. Rev. D 73, 094026 (2006).

[25] B. Adeva et al. (Spin Muon Collaboration), Phys. Lett. B 369, 93 (1996).

[26] G. Baum et al. (COMPASS Collaboration), COMPASS: A Proposal for a Common Muon and Proton Apparatus for Structure and Spectroscopy, Reports No. CERN-SPSLC96-14 and No. CERN-SPSLC-P-297, 1996.

[27] M. Alekseev et al. (COMPASS Collaboration), Phys. Lett. B 660, 458 (2008).

[28] E. Christova and E. Leader, J. Phys. Conf. Ser. 678, 012012 (2016).

[29] C. Adolph et al. (COMPASS Collaboration), Nucl. Phys. B886, 1046 (2014).

[30] G. Ingelman, A. Edin, and J. Rathsman, Comput. Phys. Commun. 101, 108 (1997).

[31] M. Asai, A. Dotti, M. Verderi, and D. H. Wright (GEANT4 Collaboration), Ann. Nucl. Energy 82, 19 (2015).

[32] P. Abbon et al. (COMPASS Collaboration), Nucl. Instrum. Methods Phys. Res., Sect. A 577, 455 (2007).

[33] H. L. Lai, J. Huston, S. Kuhlmann, J. Morfin, F. Olness, J. F. Owens, J. Pumplin, and W. K. Tung (CTEQ Collaboration), Eur. Phys. J. C 12, 375 (2000).

[34] D. de Florian, R. Sassot, and M. Stratmann, Phys. Rev. D 75, 114010 (2007).

[35] K. Augsten et al. (COMPASS Collaboration), Addendum to the COMPASS-II Proposal, d-Quark Transversity and Proton Radius, Reports No. CERN-SPSC-2017-034, SPSC-P340-ADD-1, 2018. 